anatomical characters of the double-rooted spinal nerves, which are known to be subservient to sensation as well as to motion.

There is a still more satisfactory, and indeed infallible mode of determining the truth of physiological experiments. It is merely necessary to inquire, do the apparent results contradict our experience of what takes place-the conditions being strictly the same-in man. Suppose, for instance, that after dividing the spinal cord in a frog or salamander, you touched the hind foot with a lighted paper, or heated wire; you would find that the limb so touched became violently convulsed, and you might, without further means of judging, conclude, therefore, that the animal was suffering pain. If, however, you had an opportunity of performing the same experiment under the same conditions on the human being (and this has been done in cases of injury of the spine, causing paraplegia), you would observe the same phenomena; that is to say, the convulsion of the leg on applying heat to the foot; but if you questioned the patient you would ascertain that he suffered no pain, and was even altogether unconscious that heat had been applied to his foot. The only precaution required in these cases is to be well assured that the conditions of the experiment are the same : this being attended to, we possess the means of drawing a just conclusion in a class of phenomena in which there is the greatest chance of deception, and where, indeed, some of the most distinguished physiologists of the day, neglecting thus to scrutinise the apparent results, have fallen into and have diffused the most serious errors. In fact, if vivisections are not performed in entire subordination to comprehensive views of the general laws of organisation, and if their results are not carefully compared with the phenomena displayed in the human being, they are much more likely to retard than to promote the science of physiology. A reference to the researches of Desmoulins and Magendie will afford ample confirmation of this assertion.

There is still one other object which should be carefully kept in view in the prosecution of this the most interesting branch of your studies. You should endeavour to generalise the individual facts with which this science abounds, for it is by its general laws, not by its isolated truths, that physiology has conferred such immense benefit on medicine and surgery. Take, for instance, the general law established by Hunter, that when the main artery of a limb is obstructed, the smaller vessels of the part are capable of enlarging themselves, and of thus carrying on the circulation; a principle upon which the modern operation for aueurism mainly rests. Or, again, consider the great results which have sprung from the principle established by that illustrious man whose recent loss science has to deplore, that a nervous fibre is capable of transmitting an impression in one direction only; and, consequently, that a nerve with one root has one function, and a nerve with two roots two functions. Laws like these afford endless practical applications, and they will, doubtless, be the means by which the healing art will ultimately attain a degree of exactness and efficiency, of which at present we can hardly form a just conception. $-M r$. Grainger's Lecture, Oct. 1.

\section{MALFORMATION OF TWINS.}

\section{To the Editor of The LANCET.}

SIR,-Having had an opportunity of inspecting a very unique and fanciful production of nature, perhaps the accompanying sketch and descriptive remarks may be interesting to some of your readers.

On Tuesday, the 4th ult., Mr. Turner, the gentleman with whom I am an assistant, was informed by a midwife that she had on that morning delivered a woman of twins, which were very strangely connected, and, as the parent resided in the vicinity, she recommended him to inspect them. Accordingly on that evening Mr. T. politely invited me to accompany him, and great was our surprise on beholding the biformed, two-in-one, first born, whose outlines I have delineated. The mother stated that she had gone her full period, but had not been sensible of any novement of the feetus during the last two days, which declaration was corroborated by its cuticular aspect. She had, as might have been expected, a rather protracted labour; but the midwife could not give a satisfactory account of the manner of delivery. The twins, if I may so designate the monstrous form, are rather small, the larger measuring seventeen inches longitudinally, and the other being about an inch shorter. They are broad in proportion. They are two almost perfect male children, save being united down the whole length of the bodies. There are two spinæ and two sterna, but only two breasts, the other two being evidently obliterated by the osseous union of the chests, the region intervening the approximate margins of the sterna being a bony plane. Only two of the arms are seen anteriorly, in consequence of the junction having taken place immediately before the others. There is but one cord, or funis $(f)$, with its natural complement of vessels, and it protrudes through the centre of the inferior wall of the medium of connection.

Many speculations may arise relative to the internal structure of this interesting lusus naturce, and I regret that it has not been in my power to obtain a knowledge of the minutia and modus operandi of so remarkable a machine. Mr. Turner, as well as some of his medical friends, did all in their power to induce the paternal parent to part 
with this prodigy, or allow of a post-mortem examination, but theirr efforts were ditavailirg. I am, Sir, your humble servant,

\section{Rose-place, Liverpool,}

D. MAcKINDER. Oet. 14, 1842.
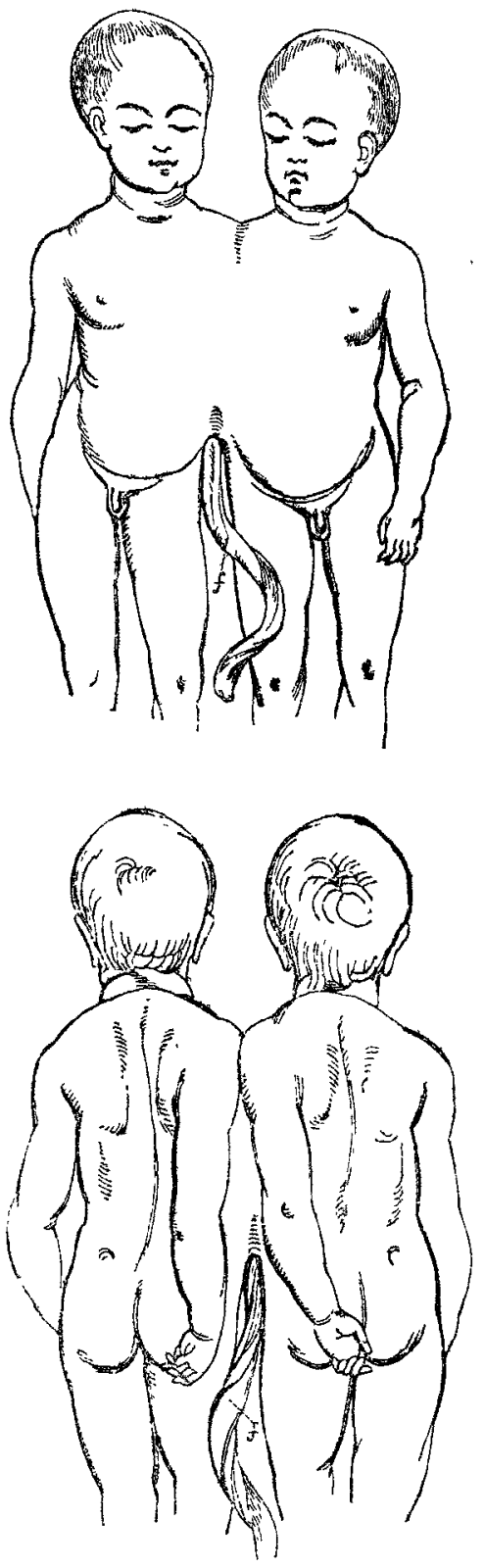

\section{DISCOVERY AND DEMONSTRA. TION OF THE NERVES OF THE UTERUS.}

Some labours on the nervous system of the uterus were commenced four years since, and have now been brought to a successful termination. Dr. W. Hunter was the first who distinctly traced the great sympathetic and sacral nerves into the uterus, and suspected that the nerves enlärged during prig. nancy. As he never examined the nerves of the unimpregnated uterus, and sawl the nerves of the gravid uterus dissected only in one subject, he could not positively assert that the nerves increased after conception. Mr. John Hunter denied that the nerves of the uterus ever enlarged during pregnancy in the smallest degree. Dr. W. Hunter left no dissected preparation of the nerves of the uterus, nor didd Mr. John Hunter, to support his conflicting statements; and in 1838 there were no preparations of the nerves of the human unimpregnated or gravid uterus, nor of the uterus of any of the lower animals in this country. In 1822, Tiedemann published a description of the nerves of the uterus, with two plates, in which only a few small branches of nerves from the great sympathetic were represented. In 1823, Lobstein stated that the uterus both before and after conception was very sparingly supplied with nerves ; and in 1829, Professor Osiander declared that the nerves of the human uterus had never been seen, and that he could only assert, from the irritability of the uterus, that it did possess nerves.

In the last volume of the "Philosophical Transactions," there is a paper on the Nervous Ganglia of the Uterus, in which it is stated, from dissections of the unimpregnated uterus, and of the gravid nterus in the third, fourth, sixth, seventh, and ninth months of preguancy, that there are many large ganglia formed on the uterine nerves, and on those of the vagina and bladder, which enlarge with the coats, blood-vessels, and absorbents of the uterus during pregnancy, and which return after parturition to their original condition before conception takes place. In the two engravings which accompany the paper, are represented these ganglia and nerves of the uterus, vagina, and bladder, in the fourth month of pregnancy. But these constitute but a small portion of the nervous system of the human uterus ; and the forthcoming volume of the "Philosophical Transactions" will contain a further accouut of the nervous structures of that organ, with an engraving, in which have been accurately represented all the great blood-vessels and nerves of the gravid uterus, on the left side, at the end of the ninth month of pregnancy. About an inch above the origin of the mesenteric artery each cord of the great sympathetic nerve sends numerous branches which form a plexus around the trunk of the artery, which accompany the ramifications of the vessel, and particularly the hæmorrhoidal artery, to the rectum. The two cords of the great sympathetic, after giving off these nerves, pass down before the aorta, about two inches below its bifurcation, where they separate, the left passing down behind the hypogastric blood-vessels to the side of the neck of the uterus, and terminating in what has been called the left utero-cervical or hypogastric ganglion. This you see is a very 